University of Nebraska - Lincoln

DigitalCommons@University of Nebraska - Lincoln

Stephen Ducharme Publications

Research Papers in Physics and Astronomy

August 1989

\title{
Absorption at radio frequencies in superconducting $\mathrm{Y}_{1} \mathrm{Ba}_{2} \mathrm{Cu}_{3} \mathrm{O}_{y}$
}

Stephen Ducharme

University of Nebraska - Lincoln, sducharme1@unl.edu

R. Durny

Department of Physics, University of Utah - Salt Lake City

J. Hautala

Department of Physics, University of Utah - Salt Lake City

D.J. Zheng

Department of Physics, University of Utah - Salt Lake City

P.C. Taylor

Department of Physics, University of Utah - Salt Lake City

See next page for additional authors

Follow this and additional works at: https://digitalcommons.unl.edu/physicsducharme

Part of the Physics Commons

Ducharme, Stephen; Durny, R.; Hautala, J.; Zheng, D.J.; Taylor, P.C.; Symko, O.G.; and Kulkarni, S., "Absorption at radio frequencies in superconducting $\mathrm{Y}_{1} \mathrm{Ba}_{2} \mathrm{Cu}_{3} \mathrm{O}_{y}$ " (1989). Stephen Ducharme Publications. 24.

https://digitalcommons.unl.edu/physicsducharme/24

This Article is brought to you for free and open access by the Research Papers in Physics and Astronomy at DigitalCommons@University of Nebraska - Lincoln. It has been accepted for inclusion in Stephen Ducharme Publications by an authorized administrator of DigitalCommons@University of Nebraska - Lincoln. 


\section{Authors}

Stephen Ducharme, R. Durny, J. Hautala, D.J. Zheng, P.C. Taylor, O.G. Symko, and S. Kulkarni 


\title{
Absorption at radio frequencies in superconducting $\mathrm{Y}_{1} \mathrm{Ba}_{2} \mathrm{Cu}_{3} \mathrm{O}_{y}$
}

Stephen Ducharme, a) R. Durny, J. Hautala, D. J. Zheng, P. C. Taylor, and O. G. Symko

Department of Physics, University of Utah, Salt Lake City, Utah 84112

S. Kulkarni

Ceramatec, Inc., Salt Lake City, Utah 84115

(Received 16 December 1988; accepted for publication 15 April 1989)

\begin{abstract}
The large magnetic-field-dependent ac absorption in superconducting $\mathrm{Y}_{1} \mathrm{Ba}_{2} \mathrm{Cu}_{3} \mathrm{O}_{y}$ ceramics and powders decreases slowly with increasing frequency in the range $2-16 \mathrm{MHz}$. The magnetic-field-dependent ac absorption is observed below $T_{c}$ in superconducting $\mathrm{Y}_{1} \mathrm{Ba}_{2} \mathrm{Cu}_{3} \mathrm{O}$ ceramics, powders and twinned crystals and in $(\mathrm{La}, \mathrm{Sr})_{2} \mathrm{CuO}_{4}$ and $\mathrm{BaPb}_{0.75} \mathrm{Bi}{ }_{0.25} \mathrm{CuO}_{3}$ powders and appears to contain distinct contributions from trapped fux and/or intragrain tunnel junctions and from ordinary superconductor surface impedance. The contribution from trapped fiux and/or intragrain tunnel junctions is important in de magnetic fields of up to approximately $20 \mathrm{G}$ at all temperatures below $T_{c}$. There is an identifiable modulated ac absorption which changes slowly over more than $12 \mathrm{kG}$ at low temperatures and which we propose is primarily caused by the ac surface impedance with possible contributions from the critical state. A twinned crystal of $\mathrm{Y}_{1} \mathrm{Ba}_{2} \mathrm{Ci}_{3} \mathrm{O}_{y}$ exhibits the same ac absorption except that it has an anisotropic dependence on the applied de magnetic field.
\end{abstract}

\section{ITRODUCTION}

The recent discoveries of superconductivity at record high temperatures in copper-octahedral oxygen perovskites ${ }^{1}$ have opened a new and exciting chapter in the field of superconductivity. While superconducting transition temperatures $^{2}$ of $125 \mathrm{~K}$ (and perhaps higher ${ }^{3}$ ) promise economical application of superconductivity on an unprecedented scale, the scientific community is striving to understand the mechanism and behavior of the new superconductors through experimentation and theoretical modeling. Many of the basic superconductivity-related properties of the new materials have been measured in recent months: the resistive transition and magnetic susceptibility, ${ }^{1}$ critical fields, ${ }^{4}$ the energy gap, ${ }^{5}$ and macroscopic quantum phenomena. ${ }^{6}$ We observed ${ }^{7}$ an unusually large field-modulated microwave absorption signal below $T_{\mathrm{c}}$ in $\mathrm{Y}_{3} \mathrm{Ba}_{2} \mathrm{Cu}_{3} \mathrm{O}_{y}$ ceramics and powders and have since observed $d^{8}$ similar modulated absorption in a twinned crystal of $\mathrm{Y}_{1} \mathrm{Ba}_{2} \mathrm{Cu}_{3} \mathrm{O}_{4}$ and in $\left(\mathrm{La}_{3} \mathrm{Sr}_{2} \mathrm{CuO}_{4}\right.$ and $\mathrm{BaPb}_{1-x} \mathrm{Bi}_{2} \mathrm{O}_{3}$ (BPB) powders. The modulated absorption can be separated qualitatively and quantitatively into distinct contributions from the ac surface impedance observed in all superconductors plus a new contribution observed only at low dc field $(\leqslant 20 \mathrm{G})$ in the perovskite superconductors. In Ref. 7 we showed that the field-modulated ac absorption signal in $\mathrm{Y}_{1} \mathrm{Ba}_{2} \mathrm{Cu}_{3} \mathrm{O}_{y}$ at $9.4 \mathrm{GHz}$ appears abruptly at $T_{c}$ as a sample is cooled and increases steadily to a constant value at low temperatures. The modulated absorption signal at a fixed temperature decreases as a dc magnetic feld is applied, decreasing rapidly over approximately $20 \mathrm{G}$ (the contribution which appears unique to perovskite superconductors) and then more slowly over several $k G$. We now report the frequency dependence of the field-modulated ac absorption component of the macroscopic ac magnetic sus-

\footnotetext{
"Present address: IBMM Amaden Research Center, San Jose, CA 95120.
}

ceptibility in superconducting $\mathrm{Y}_{1} \mathrm{Ba}_{2} \mathrm{Cu}_{3} \mathrm{O}_{\text {y }}$ powders measured at temperatures from $20 \mathrm{~K}$ to $T_{c}$ and frequencies 2-16 $\mathrm{MHz}$ as well as at $9.4 \mathrm{GHz}$. We observe a similar modulated ac absorption at $9.4 \mathrm{GHz}$ at low fields in superconducting ceramics, powders, and twinned crystais of $\mathrm{Y}_{1} \mathrm{Ba}_{2} \mathrm{Cu}_{3} \mathrm{O}_{y}$ and in superconducting powders of $\left(\mathrm{La}_{3} \mathrm{Sr}\right)_{2} \mathrm{CuO}_{4}$ and $\mathrm{BPB}$.

In the next section we describe how the ceramic samples were made and prepared. The following section describes the radio and microwave frequency measurements. There follows a detailed description of the behavior of fieid-modulated ac absorption under a variety of conditions: varying temperature, dc magnetic field, sample quality, and measurement frequency. We conclude with a discussion of several possible mechanisms and further details which should be included in a complete model of the ac absorption in high-temperature superconductors.

\section{SAMPLE PREPARATION}

The superconducting $\mathrm{Y}_{1} \mathrm{Ba}_{2} \mathrm{Cu}_{3} \mathrm{O}_{y}$ ceramic samples were prepared by solid-state reaction of $\mathrm{BaCO}_{3}$ (reagent grade), $\mathrm{Y}_{2} \mathrm{O}_{3}$ (99.999\%), and $\mathrm{CuO}$ (puratronic grade). All powders were mixed and ground for $24 \mathrm{~h}$ with ethanol and alumina. Mixed powders were dried and fred in an alumina crucible at $940^{\circ} \mathrm{C}$ in air for $50 \mathrm{~h}$. Subsequently, calcined powder was milled for $24 \mathrm{~h}$ and binder added. Pellets with $1.5 \mathrm{~cm}$ diameter and $0.3 \mathrm{~cm}$ thickness were pressed from the powder-binder mixture at 35 psi. The pellets were fired at $970^{\circ} \mathrm{C}$ for $24 \mathrm{~h}$ and cooled at a rate of $50^{\circ} \mathrm{C} / \mathrm{h}$. (The binder was completely removed during sintering.) The pellets were then annealed in oxygen at 15 psi at $950^{\circ} \mathrm{C}$ and then cooled at a rate of $30^{\circ} \mathrm{C} / \mathrm{h}$.

For the resistance measurements, leads were attached to the ceramic pellets with silver paint in a four-terminal con- 
figuration. The $R(T)$ curves were not measurably affected by currents up to $0.2 \mathrm{~A} / \mathrm{cm}^{2}$.

Powdered samples were obtained by coarsely grinding ceramic pieces (e.g., particles range from $<1 \mu \mathrm{m}$ to several dozen $\mu \mathrm{m}$ in size). The twinned crystal was selected from the powder and glued to the end of a quartz rod. The samples studied by ac absorption, ceramic pieces, powders, or the twinned crystal, were placed in a $2 \mathrm{~mm}$ diameter $\times 250 \mathrm{~mm}$ long ESR grade quartz tube. The samples were fushed and flled with helium gas at atmospheric pressure, and sealed to prevent sample deterioration from contact with oxygen and water vapor.

\section{HI. EXPERIMENTAL TECHNQUE}

The modulated ac absorption measurements were made with the two different types of apparatus described below. The modulated absorption signal is conceptually the same in both types of apparatus. The sample was subjected to three magnetic fields: a do field which determines the state of the sample (at ixed temperature); a low-frequency ( $350 \mathrm{~Hz}$ or $100 \mathrm{kHz}$ ) field which modulates the sampie state a small amount about the dc state; and a high-frequency $(2 \mathrm{MHz}-$ $9.4 \mathrm{GHz}$ ) probing field which induces a magnetic response in the sample which was detected as a function of the lowerfrequency modulation.

The microwave $(9.4-\mathrm{GHz}$ ) modulated ac absorption measurements were made in the tuned resonant cavity (Fig. [(a)] of a Briker ER200D-SRC ESR spectrometer and the measurements made in the same manner as crdinary ESR spectra except that in this case the measured absorption is not from a magnetic resonance. The quartz tube containing the superconducting sample was placed in a position of max-

(a)

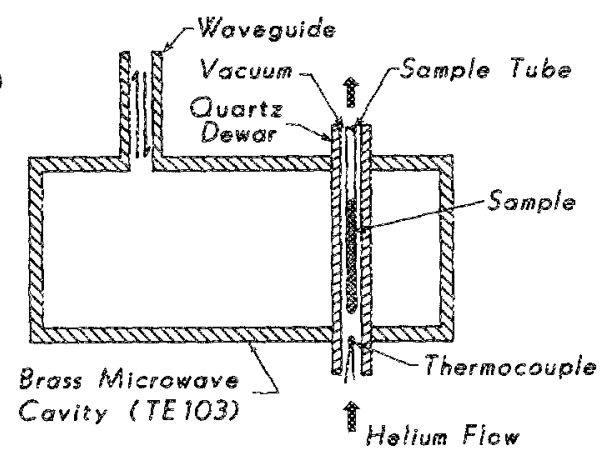

(b)

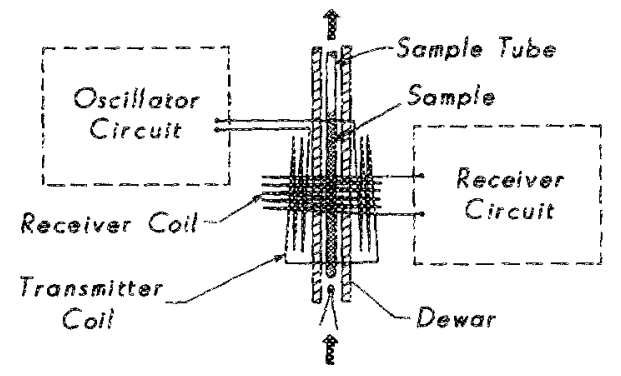

FIG. 1. Diagram of the ac susceptibility apparatus: (a) Microwave resonant cavity and helium flow cryostat used at $9.4 \mathrm{GHz}$. (b) Matched rf coils and helium fow cryostat used in the range $2-16 \mathrm{MHz}$. imum microwave magnetic field (minimum electric field) in the resonant cavity [see Fig. 1(a)] which was critically coupled (minimum refiectivity) to the input/output waveguide. A dc magnetic feld plus a small ac modulation field (at 100 $\mathrm{kHz}$ ) were applied and the modulated part of the reflected power from the resonant cavity was detected by phase-sensitive amplification at the frequency of the modulation field. The demodulated signal was then digisized and recorded by a computer. The field modulation technique used gives rise to a derivativelike signal proportional to the imaginary part (absorption) of the macroscopic ac magnetic susceptibility of the sampie when the modulation amplitude exceeded approximately $1 \mathrm{G}$. Lower modulation amplitudes resulted in dramatically different results, depending on the direction of the dc field scan.

The radio frequency (rf) modulated absorption measurements were performed with the same helium flow cryostat but with a different ac susceptometer. Figure 1(b) shows the arrangement of the superconducting sampie and twin if coils of the Varian model V-4200B NMR spectrometer. The rf waves were generated by a tuned circuit containing the "transmitter" coil and detected by an identical "receiver" coil and circuit oriented nearly orthogonal to the "transmitter" coil. The received power was then proportional to the mutual inductance of the coils. The (macroscopic) ac magnetic susceptiblity of the sample placed within the coils [Fig. $l(b)]$ altered their mutual inductance. The relative phase of the "transmitter" and receiver circuits could be adjusted so that either the in-phase (dispersion) or out-ofphase (absorption) components of the macroscopic ac magnetic susceptibiity could be measured. A small ae modulation magnetic field $(0.2-2.0 \mathrm{G}$ at $355 \mathrm{~Hz})$ plus a de magnetic field $(0-12 \mathrm{kG})$ was applied to the sample and the moduiated (at $355 \mathrm{~Hz}$ ) part of the receiver power detected by phasesensitive amplification and recorded by a computer as in the microwave absorption measurements. The modulated ac absorption signal in the rf apparatus includes both magnetic and electric contributions, unlike the microwave modulated absorption signal which inciudes magnetic contributions only. Because of the similarity of the radio and microwave frequency signals, we assume they are both magnetic in or:gir.

In both the microwave and $r$ ac absorption measurements, a standard ESR resonant medium (DPPH) was used to calibrate the magnitude of the absorption measured in the superconductor. This is particularly important in order to compare the signal strength at different frequencies as the mutual inductance and detector efficiency of the rf coils are a strong function of frequency. Care was taken to minimize the effect of the sample on coil or cavity tuning.

Temperature control during the ac absorption measurements was accomplished with an Air ${ }^{3}$ roducts Heli-Tran helium flow cryostat with calibrated chromel-gold $0.07 \%$ Fe) thermocouples and microprocessor feedback to a heater mounted in the helium fow [see Fig. 1(a)]. Since the thermocouple was outside the sample tube, a small systematic error in temperature was unawoidable; the superconducting sample was generally a few degrees warmer than the thermocouple mounted upstream in the helium gas flow. 
Because of the number and variety of reports of similar but different measurements of ac absorption in perovskite superconductors the casual reader, and perhaps even the expert, may have trouble sorting out the similarities and the differences. The most common observation reported is that of a "zero-field" absorption signal obtained with a field modulation technique in an electron-spin-resonance apparatus like the Brüker ESR spectrometer used in our microwave frequency measurements. Published reports of this nature of which we are aware are Refs. 7-13 though many new reports appear in the literature each month. The use of an ESR spectrometer benefts from extremely high sensitivity but the large diamagnetic dispersion response of superconducting samples can produce false absorption signals as pointed out in Ref. 10 .

A second, similar techrique in studying the ac magnetic response of superconductors is measurement of the frequency shift (dispersion) and quality factor (absorption) of a resonant cavity, ${ }^{13-16}$ or of a waveguide ${ }^{17}$ containing a superconducting sample without an additional modulation magnetic field as used in ESR spectrometers.

The techniques involving ESR spectrometers have some important variations. Modulation amplitude appears to be the most important parameter; Portis et al., ${ }^{13}$ Blazey et al.,

and Durny et al. ${ }^{18}$ report a dramatically different modulated signal, which depends antisymmetrically on the direction of the de field scan, observed at small modulation amplitudes. The radio frequency measurements reported here are at relatively large modulation amplitudes, $\frac{1}{2} \mathrm{G}$, and show no hysteresis or dependence on the scan direction. For this and other reasons to be discussed, we observe that modulation tech miques generally do not allow the superconducting samples to be in equilibrium.

\section{W. RESULTS}

We will first characterize the behavior of the ac absorption and show that it includes a contribution at low de fields unique to the high $T_{c}$ perovskite superconductors, behaving differently from the commonly observed "skin effect" surface impedance (absorption) due to conduction electrons. First the observed ac absorption has a pronounced dependence on the magnitude of an applied dc magnetic field. Figure 2 shows typical absorption signals observed in superconducting $\mathrm{Y}_{1} \mathrm{Ba}_{2} \mathrm{Cu}_{3} \mathrm{O}$, powder at $6 \mathrm{MHz}$. There are two distinct signals antisymmetric about zero magnetic field. The first is a relatively narrow "derivativelike" signal [Fig. 2 (a) ] with a width of approximately $10-30 \mathrm{G}$, depending on

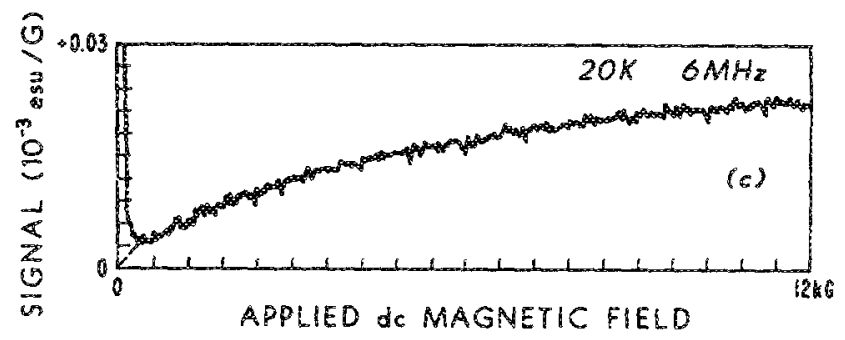

(a)
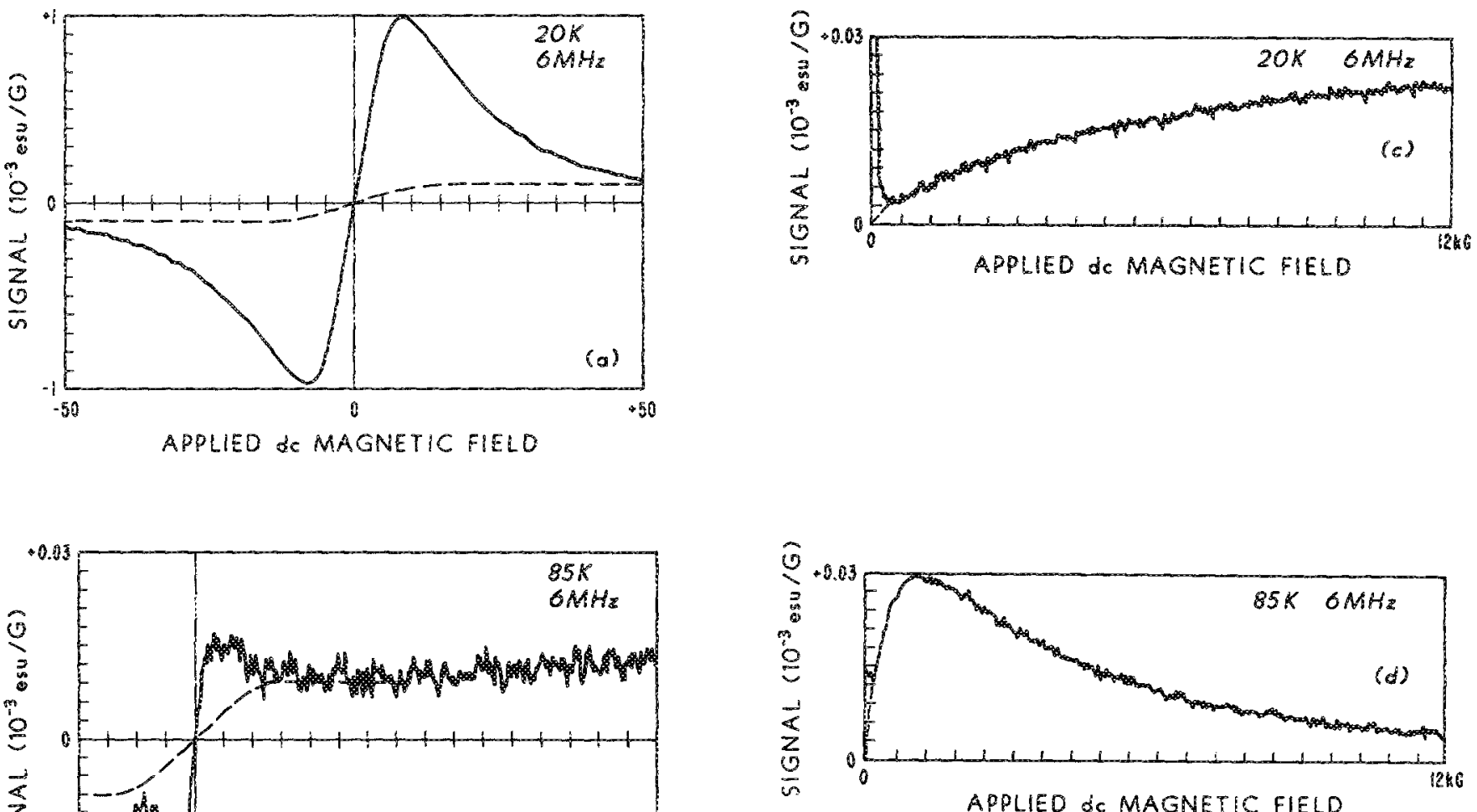

(b)

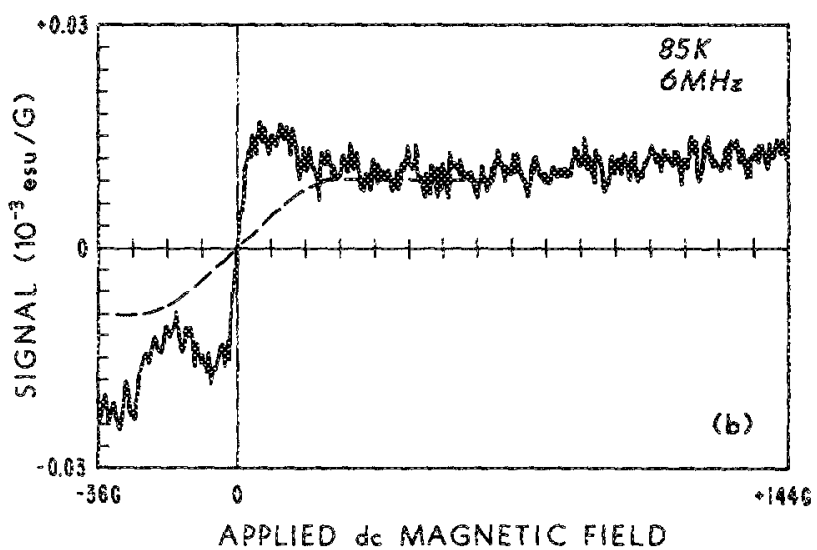

FIG. 2. Magnetic field dependence of the modulated ac absorption in $\mathrm{Y}_{1} \mathrm{Bba}_{2} \mathrm{Cu}_{3} \mathrm{O}_{y}$. (a) At $20 \mathrm{~K}$ between -100 and $+100 \mathrm{G}$. (b) At $85 \mathrm{~K}$ from -36 io $144 \mathrm{G}$. (c) and (d) From 0 to $1.2 \mathrm{~T}$ at 20 and $85 \mathrm{~K}$, respectively. The dotted lines show the approximate contribution from the surface impedance. 


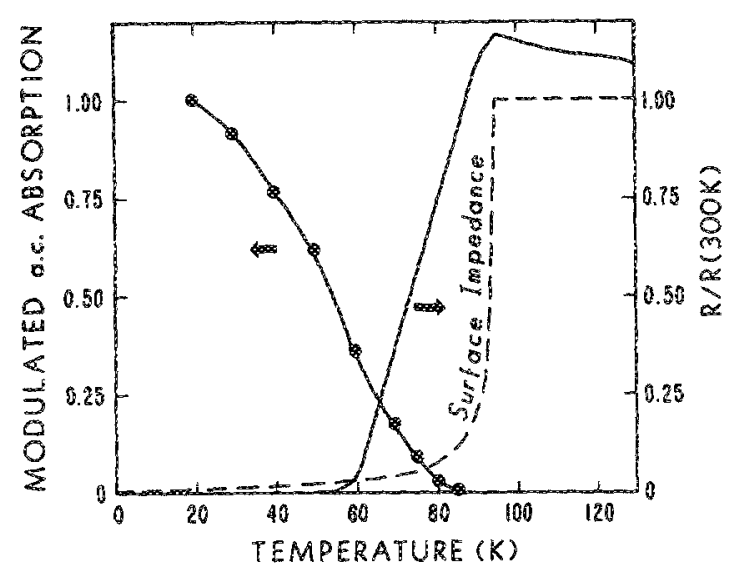

FIG. 3. Temperature dependence of the modulated ac absorption signal at 6 $\mathrm{MHz}$ (relative to the signal at $20 \mathrm{~K}$ ) and the de resistivity (relative to the resistivity at $300 \mathrm{~K}$ ). The usual surface impedance is shown schematically by the dashed line.

temperature and frequency. The narrow "signal" sits on top of a very broad signal indicated by the dotted line in Fig. $2(a)$. As the temperature approaches $T_{c}$ from below the narrow contribution to the modulated absorption decreases more rapidly than the broad contribution as is evident in comparing Figs. $2(a)$ and $2(b)$. The results and discussion which follow refer to the narrow, or low-field, nodulated absorption signal unless otherwise noted. The ac dispersion measurements from 2 to $16 \mathrm{MHz}$ result in a signal similar to those in Fig. 2 except that the dispersion has twice the width and half the magnitude (relative to the standard DPPH) of the modulated ac absorption.

The pronounced temperature dependence of the narrow modulated absorption is shown in Fig. 3. The ac absorption appears only at temperatures below $T_{c}$, the superconducting transition temperature, and increases steadily to a large, conv stant, value at low temperatures. ${ }^{7}$ The modulated absorption appears concurrently with presence of the superconducting state. The high sensitivity of the modulated absorption measurements (especially at $9.4 \mathrm{GHz}$ where the resonant cavity one of used has a dynamic range of at least five decades) greatly exceeds that for resistive or Meissner fux exclusion measurements near $T_{c}$. The modulated ac absorption is therefore one of the most sensitive measure of $T_{\mathrm{c}}$ presently available in ceramics and twinned crystals. ${ }^{72}$. The temperature dependence of the ac surface impedance ${ }^{16}$ ("skin-effect") in a superconducting $\mathrm{Y}_{1} \mathrm{Ba}_{2} \mathrm{Cu}_{3} \mathrm{O}_{y}$ is also shown in Fig. 3 for comparison. The surface impedance in $Y_{1} \mathrm{Ba}_{2} \mathrm{Ca}_{3} \mathrm{O}_{y}$ is weaker than the surface impedance in most metals due to the low carrier density of about $10^{21} \mathrm{~cm}^{-3}$. Another indication of the unique, superconductivity-related character of the ac absorption is the dependence on sample quality. The modulated ac absorption signal, as well as the observed Meissner fraction, increases with the increasing proportion of superconducting $\mathrm{Y}_{1} \mathrm{Ba}_{2} \mathrm{Cu}_{3} \mathrm{O}_{y}$ in the sample. Even samples with $100 \% \mathrm{Y}_{1} \mathrm{Ba}_{2} \mathrm{Cu}_{3} \mathrm{O}_{y}$, the strength of the ac absorption may still vary with slight variations in prepo aration conditions.

Powders of $\mathrm{La}_{1.9} \mathrm{Sr}_{0.1} \mathrm{CuO}_{4}$ and $\mathrm{BaPb}_{0.75} \mathrm{Bi}_{0.25} \mathrm{O}_{3}$ (BPB) showed similar modulated absorption signals at 9.4
GHz as shown in Figs. 4(a) and 4(b). (The signals were too weak to measure with the radio frequency apparatus.) The modulation amplitude for both spectra was $2.5 \mathrm{G}$ which was sufficiently large to produce an antisymmetric signal in $\mathrm{La}_{1,9} \mathrm{Sr}_{0,1} \mathrm{CuO}_{4}$ but not in $\mathrm{BPB}$. The asymmetry observed in Fig. 4 (a) is similar to that observed in $\mathrm{Y}_{1} \mathrm{Ba}_{2} \mathrm{Cu}_{3} \mathrm{O}_{3}$ sample under similar conditions but with modulation ampitude $\sim 1 / 4 \mathrm{G} .{ }^{18}$ The temperature dependence of the magnitude of the modulated absorption signal in powdered $\mathrm{La}_{1.85} \mathrm{Sr}_{0.15} \mathrm{CuO}_{4}$ is shown in Fig. $4(\mathrm{c})$.

The frequency dependence of the modulated ac absorption in a powder of $\mathrm{Y}_{1} \mathrm{Ba}_{2} \mathrm{Ca}_{3} \mathrm{O}_{y}$ is shown in Fig. 5. The
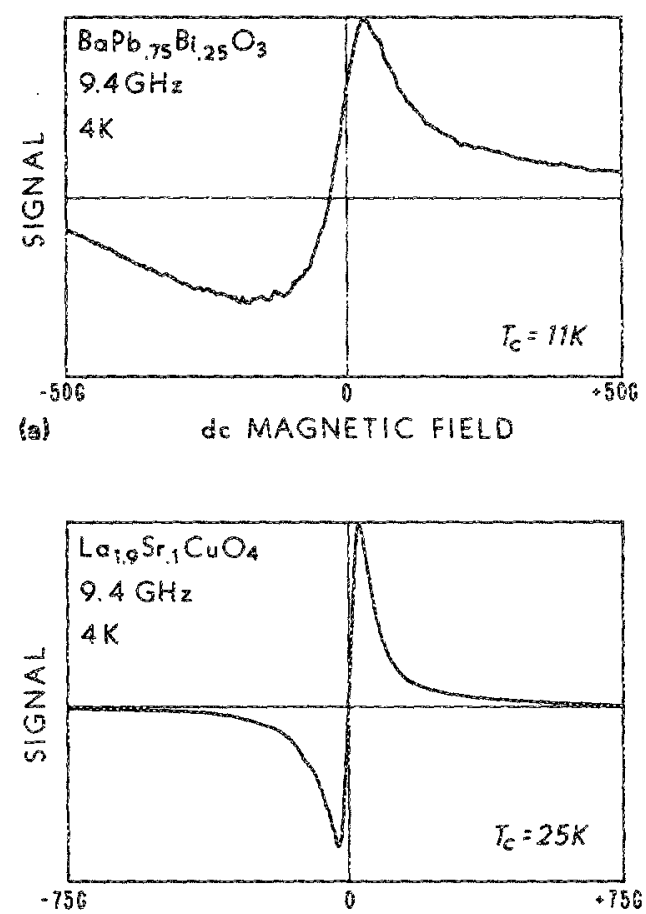

(b)

de MAGNETIC FIELD

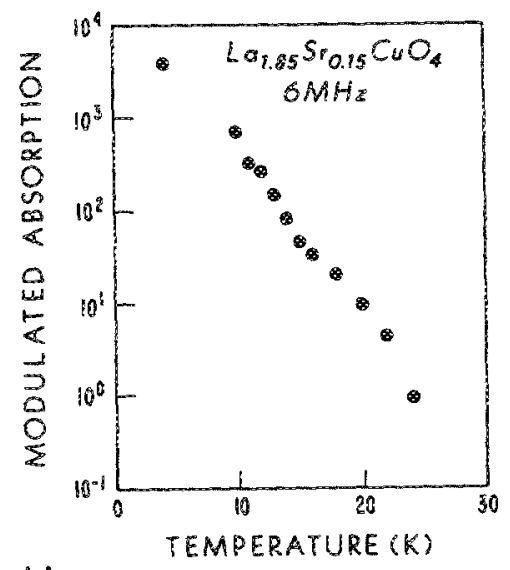

set

FIG. 4. Modulated ac absorption signals at $9.4 \mathrm{GHz}$ at $4 \mathrm{~K}$ in (a) $\mathrm{HaPb}_{n, 75} \mathrm{Ri}_{0.25} \mathrm{O}_{3}\left(T_{c} \approx 11 \mathrm{~K}\right)$ with modulation ampiiude $2.5 \mathrm{G}$ and $(\mathrm{b})$ $\mathrm{La}_{1.9} \mathrm{Sr}_{\mathrm{G} .1} \mathrm{CuO}_{4}\left(T_{c} \approx 25 \mathrm{~K}\right)$ with modulation amplitude $2.5 \mathrm{G}$. (c) Magnitude of the modulated absorption in $\mathrm{La}_{4.85} \mathrm{Sr}_{0.15} \mathrm{CuO}_{4}$ powder vs temperature. 


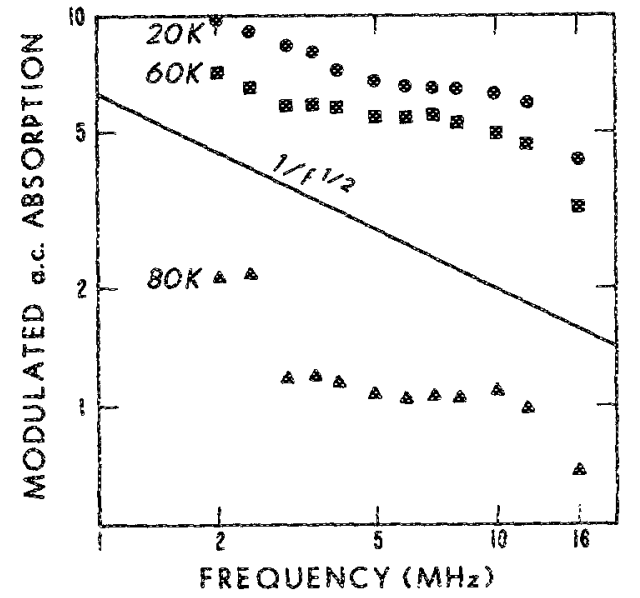

FIG. 5. Frequency dependence of the modulated ac absorption from 2 to 16 $\mathrm{MHz}$ at three different temperatures. The solid line is the function $f^{-1 / 2}$ shown for comparison.

radio frequency $(2-16 \mathrm{MHz})$ measurements were made using the nearly orthogonal tuned coils as described above [Fig. 1(b)]. The modulated absorption at a fixed tempera. ture decreases as the frequency increases. The solid line has a slope of $-\frac{1}{2}$ and is slightly steeper than the data. The modulated absorption (narrow peak) at $9.4 \mathrm{GHz}$ at $20 \mathrm{~K}$ is approximately 40 times weaker than at $10 \mathrm{MHz}$ for an overall change of 1.7 decades with a frequency change of nearly 4 decades. The surface impedance, ${ }^{15,16}$ in contrast, increases approximately as $f^{2}$.

Figure 6(a) shows the temperature dependence of the modulated absorption strength and the modulated dispersion signals at several frequencies. The width of the narrow modulated absorption and dispersion signals at different frequencies are shown in Fig. $6(\mathrm{~b})$.

Though the ac absorption (see Fig. 3) is not significantly diferent in superconducting ceramic pieces or powders, when a crystal which is presumably twinned is selected from the powder the observed signal exhibits two distinctly different peaks depending on how the sample is oriented with respect to the applied dc magnetic field. A $100 \times 100 \times 10 \mu \mathrm{m}^{3}$ twinned crystal plate of $\mathrm{Y}_{1} \mathrm{Ba}_{2} \mathrm{Cu}_{3} \mathrm{O}_{y}$ was mounted with the $c$-axis perpendicular to the resonant cavity axis [Fig. 1(a) 1 . The microwave absorption was measured for different orientations of the applied dc magnetic field. The narrow ac absorption signature appeared as two peaks with different width (Fig. 7). When the applied feld was oriente paralle! to the crystal $c$ axis (also perpendicular to the plate), a signal with $8 \mathrm{G}$ width was observed while when the applied fieid was oriented in the $a-b$ plane (also the plane of the plate), the width was about $40 \mathrm{G}$. (The sample was cooled in a field of approximately $25 \mathrm{G}$, the residual field of iron-core magnet used, and this may have resulted in increased overall line widths. ${ }^{18}$ ) A powder or ceramic piece exhibits a signal which is the rotational average of these two signals, about $20 \mathrm{G}$ wide (when cooled in a $25-G$ field). There are three possible sources of the uniaxial symmetry shown in Fig. 7 . The first possibility is that the absorption is uniaxial in the crystal
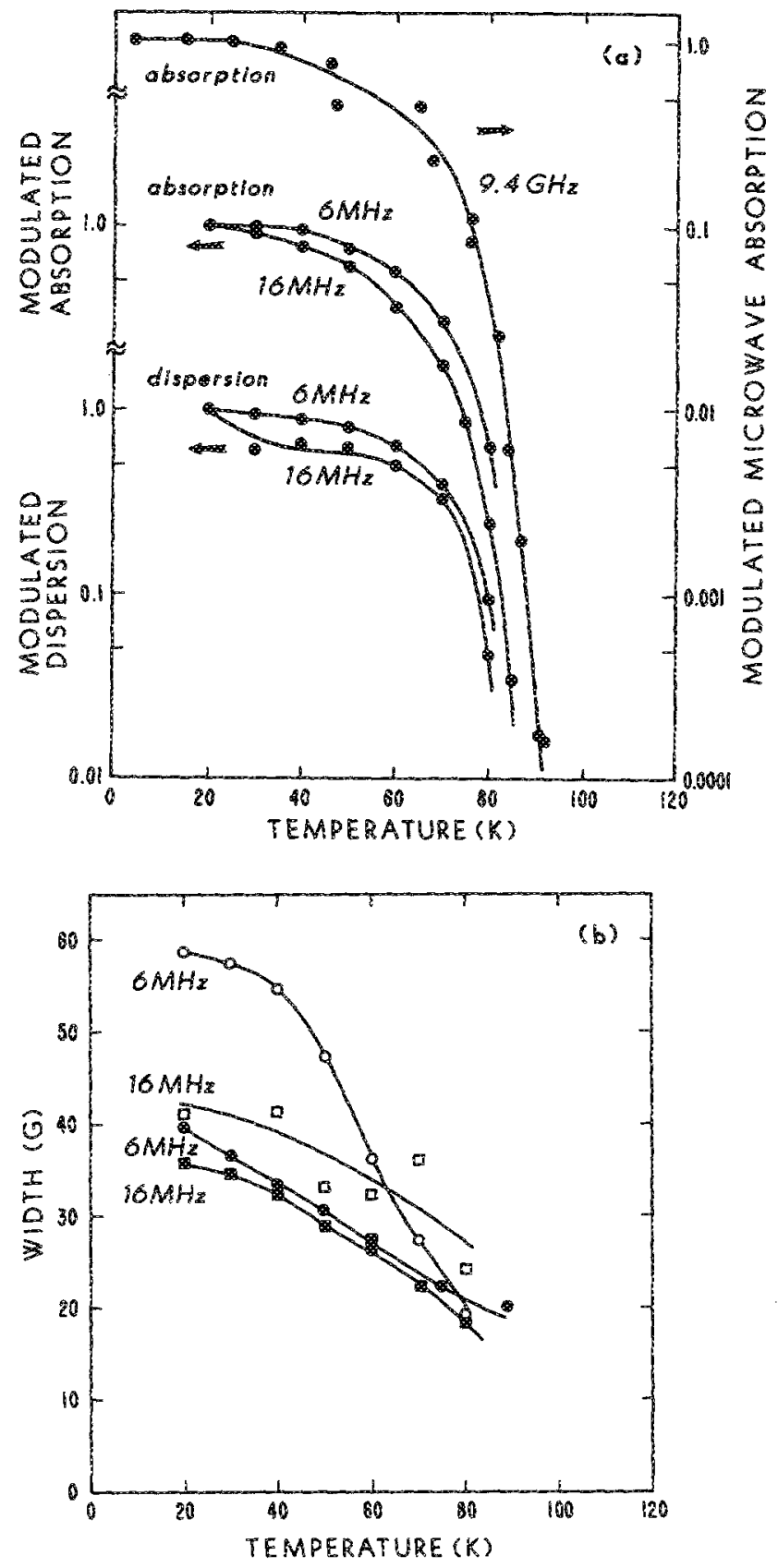

FIG. 6. Temperature dependence of the magnitude (a) and width (b) of the modulated ac dispersion and absorption at diferent frequencies. The data at each frequency are individually normalized to the signal at $20 \mathrm{~K}$. The solid lines are guides to the eye. The open (closed) symbois in (b) represent the dispersion (absorption) signals.

lattice orientation (i.e., with respect to the $c$ axis). ${ }^{19}$ The crystal geometry could also account for the two peak widths; when the applied freld is perpendicular to the plate, fux is more readily forced into the sample even at fields below $H_{c l}$. The third possibility is the anisotropy which would appear if twinning planes affect the ac absorption. But twinning planes are usually oriented perpendicular to crystal plates and would show a different anisotropy axis than that observed. We do not know if the sample studied has twinning planes, or how they are oriented, but reports of studies on many crystals of $\mathrm{Y}_{1} \mathrm{Ba}_{2} \mathrm{Cu}_{3} \mathrm{O}_{y}$ deduce that the twin planes are preferentially oriented perpendicular to a plate. ${ }^{20}$ 


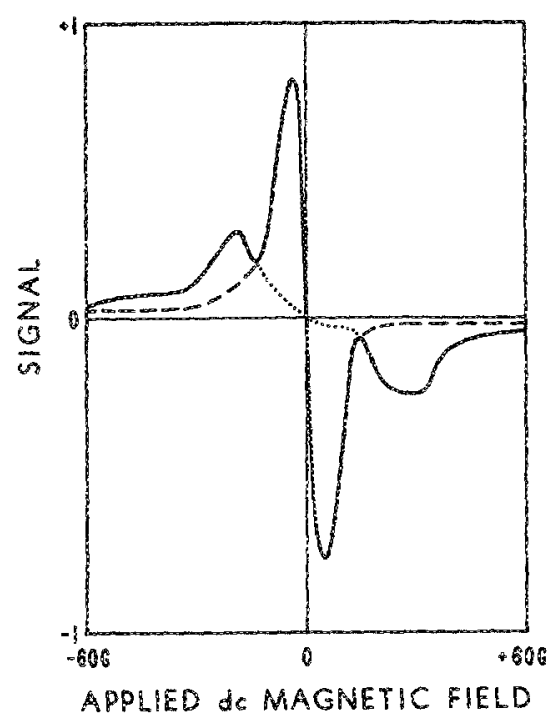

FIG. 7. Uniaxial ac absorption signal of a twimed erystal plate $\left(100 \times 100 \times 10 \mu \mathrm{m}^{3}\right)$ at $4 \mathrm{~K}, 9.4 \mathrm{GHz}$. The narrowed $(-8 \mathrm{G})$ signal appeared only when $F \perp$ plate and the wide $(-40 G)$ peak primarily when $H !$ plate. The modulation amplitude was $2 \mathrm{G}$.

Both the crystal and the powders exhibit significant hysteresis and field-cooling effects, as was observed by Müller et al. ${ }^{21}$ in $(\mathrm{La}, \mathrm{Sr})_{2} \mathrm{CuO}_{4}$ in the low-frequency magnetic susceptibility. Our observations ${ }^{18}$ show that cooling in fields as smanl as a few Gauss causes the narrow absorption signal [Fig. 2(a)] to broaden and shift its center to a nonzero applied field (e.g., fiux is trapped in the powders or ceramic pieces during cooling). The shift in the signal center is then a direc measure of the amount of trapped flux and the broadening a measure of the nonuniform fields in the sample due to the trapped fux. A similar effect is obtained even in a sample cooled in zero field if de magnetic field of a few $\mathrm{kG}$ or more is applied at low temperature and then removed. ${ }^{21-23}$ The effects of cooling $\mathrm{Y}_{1} \mathrm{Ba}_{2} \mathrm{Cu}_{3} \mathrm{O}_{y}$ in a field or applying a large field at low temperatures occur only below a "reversibility" temperature ( $T *$ in Ref. 21 ) of about $80 \mathrm{~K}^{18}$

Two other observations correlate well with the modulated ac absorption measurements. $A$ dc voltage up to a few dozen microvolts appeared across ceramic samples of $\mathrm{Y}_{1} \mathrm{Ba}_{2} \mathrm{Cu}_{3} \mathrm{O}_{y}$ when irradiated with 9.4-GHz microwaves but only at temperatures below $T_{c} .{ }^{24}$ The dc voltage scaled linearly with microwave power up to a characteristic saturation power and decreased to one-half its zero field value at about $20 \mathrm{G}$, similar in shape to the integral of Fig. 2(a). We suspect the induced voltage may be the result of ac Josephson rectification by the many grain boundaries and the twins within each grain but added together randomly due to the random phases of the individual junctions. The modulated ac absorption signal width would then correspond to the distribution of critical fields of the junctions, not the bulk critical field of the material. The second observation related to Josephson junctions is in the behavior of the critical current of a thin bridge of $\mathrm{X}_{1} \mathrm{Ba}_{2} \mathrm{Ca}_{3} \mathrm{O}_{4}$ ceramic in de magnetic fields and microwave radiation. The critical current decreases rapidly as a magnetic field is applied, again with a width of about $20 \mathrm{G}$ as in Fig. 2(a), but with faint oscillations like those observed in a single Josephson junction ${ }^{25}$ smeared out due to the presence of many junctions.

\section{DISCUSSION}

There are several characteristics of the unusually large modulated ac absorption which should be taken into consideration when evaluating potential mechanisms: (1) the sensitivity to small magnetic fields, (2) the precipitous increase in the magnitude of the modulated absorption below $T_{c}$, and (3) the frequency dependence. The other observations described in the previous section: uniaxial signals observed in a twinned crystal, hysteresis in a magnetic field, and evidence for ac Josephson effects are important clues to the mechanism of ac absorption. We will now discuss four distinct mechanisms for the modulated ac absorption and weigh their predictions against our observations. These mechanisms are dissipation in an ensemble of current loops with glassy behavior, fuxons in long Josephson junctions, pinned flux tubes in the critical state, and surface impedance.

Broad resistive transitions observed in the high $T_{c}$ ceramics have frequently been attributed to various mechanisms, one of them being glassy behavior among weakly coupled grains. ${ }^{1,2 \pi, 26}$ It is natural to interpret other observed properties in terms of a glassy state: magnetic hysteresis, ${ }^{2 l}$ the field dependence of critical currents, ${ }^{6,27}$ and the modulated ac absorption. ${ }^{23}$

Nonequilibrium behavior of disordered superconductors has often been attributed to a glassy state. The observed modulated ac absorption could also be described by a glassy state if it includes a distribution of current loops or paths within or between the twinned grains and includes a dissipation mechanisn such as intragrain Josephson junctions which break up the current loops (Fig. 8). Just such a model has been proposed by Deutscher and Müller ${ }^{27}$ in which the junctions with a superconductor-insulator-superconductor (SIS) structure consisting of a tetragonal insulating layer Which is a single unit cell in width separating the misoriented orthorhombic superconducting material. The key assumption made in order to make this structure a true SIS junction is that the superconducting energy gap in the orthorhombic

\section{GLASS STATE}

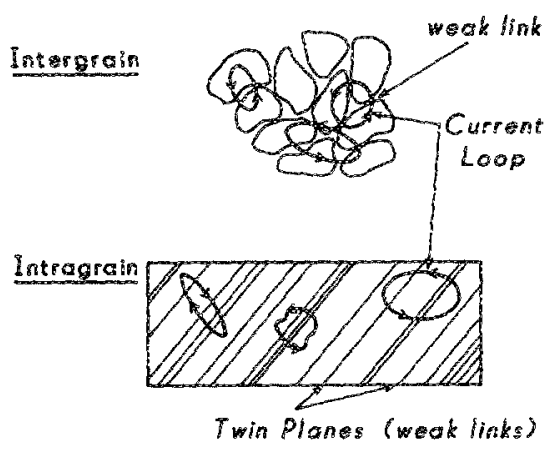

FIG. 8. Schematic diagram of current loops for a model of intergrain or intragrain glassy behavior. 
material decreases dramatically at the S-I interfaces, thus producing a tunneling barrier. This process is possible, these authors propose, ${ }^{27}$ because of the short superconducting coherence length ( lattice parameter) observed in the high $T_{c}$ superconductors. At low temperatures the gap at the S-I interface would return to the bulk value $\Delta(0)$ by the proximiity effect ${ }^{28}$ and hence the number of junctions would be reduced ( see also Fig. 1 of Ref. 27). The modulated ac absorption, in contrast, does not decrease at low temperatures, but rises to an approximately constant value as shown in Figs. 3 , 4, and 6. The requisite junctions, then, cannot be derived from the boundary-gap depression model, ${ }^{27}$ but must arise in another manner. The model based upon a superconducting glassy state proposed in Ref. 27 could still apply, together with the existence of a "geometric critical fieid" $H_{* 1}^{*}$ $=\phi_{0} / 2 s$, where $\phi_{0}$ is the fux quantum $h c / 2 e$ and $s$ is a typicall loop area.

The temperature, frequency, and field dependencies are qualitatively consistent with this type of multiple-loop glassy behavior. As discussed in Ref. 27, a superconducting glassy state with typical current loop areas comparable to the grain size ( square micrometer) would have a critical field $H_{c 1}^{*}$ $\approx 10 \mathrm{G}$ which is consistent with our measurements of the "width" of the modulated absorption feature (Fig. 6). The abrupt onset of the modulated absorption at $T_{\mathrm{r}}$ requires that the loss mechanism be associated with the superconducting state. Finally, the frequency dependence (Fig. 5) is qualitatively consistent with losses in a superconducting glassy state $^{26}$; as the frequency increases, the larger current loops have too much self-inductance and cease to follow the driving feld so that the overall absorption would decrease.

Let us assume that there are indeed Josephson junctions within each grain. Then a mechanism we propose ${ }^{8,18,24,29}$ for the modulated ac absorption is the dissipative motion of Josephson fuxons in the junctions. Portis and Blazey have worked out just such a model. ${ }^{30}$ This model is substantiated by our observations and measurements: microwave rectification by a bulk ceramic sample, magnetic field dependence of the critical current, and anisotropy of the modulated absorption in a twinned crystal. We observe ${ }^{24}$ microwave-induced voltages of about a microvolt per milliwatt of micro* waye power irradiating a bulk superconducting ceramic sample. Also, the superconducting critical current of a small $\left(1 \times 1 \times 1 \mathrm{~mm}^{3}\right)$ bridge has approximately the same magnetic field dependence as that of the modulated ac absorption [Fig. 2(a)]. Note that the modulated ac absorption, microwave rectification, and the critical currents all decrease dramatically in magnetic fields of approximately $20 \mathrm{G}$. The sensitivity to small magnetic fields [below $H_{c \text { f }}$ (Ref. 19)] is consistent with the presence of Josephson junctions within the twinned crystals. Many such junctions can have one of their dimensions larger than the Josephson penetration depth, thus allowing the creation of quantized vortices by the external magnetic field. Induced ac currents through the junction can lead to Lorentz forces on the vortices and dissipation as well as many other interesting phenomena.

The third mechanism for the modulated ac absorption is dissipation by pinned fux tubes independently, or collectively in the critical state. When cooling samples in small mag- netic fields, we observe that the modulated absorption signal is measurably altered when the magnitude of the field applied during cooling exceeds a few Gauss. When samples are cocled in fields larger than a few Gauss, the modulated $a b$ sorption signal is observed to weaken and broaden signiflcantly ${ }^{18}$ so that the magnetic fields of fux tubes affects the source of the very low-field modulated absorption if only by providing a highly inhomogeneous magnetic field. ${ }^{26}$ But the flux tubes themselves, whether individually or collectively, may be absorbing the incident radiation as the individual pinned vortices sit in a damped potential well and would absorb at all frequencies. The model proposed by Portis et al. ${ }^{13}$ accounts for collective absorption by fluxons in the critical state at fields above a few Gauss.

Yeshurun and Malozemoff ${ }^{31}$ have proposed that the do susceptibility and hysteresis in the high $T_{c}$ superconductors may be attributed to the critical state, the nonequilibrium fiux state of a "hard" type-II superconductor. ${ }^{32}$ The principle features of critical state electrodynamics ${ }^{32}$ are only partially consistent with our results though there is sufficient room for reconciling the apparent discrepancies: (1) The ac absorption in the critical state is constant above a low threshold frequency but below the gap, contrary to the slow decrease of the modulated absorption observed (Fig. 5) over a limited range of frequencies. (2) The ac absorption in the critical state should begin above the lower buik critical field $H_{c 1}$, about $50 \mathrm{G}(500 \mathrm{G})$ along the $c(a, b)$ axis' in $\mathrm{Y}_{1} \mathrm{Ba}_{2} \mathrm{Cu}_{3} \mathrm{O}_{y}$ and may indeed contribute to the absorption at higher fields (see Fig. 9). Therefore, we propose that the
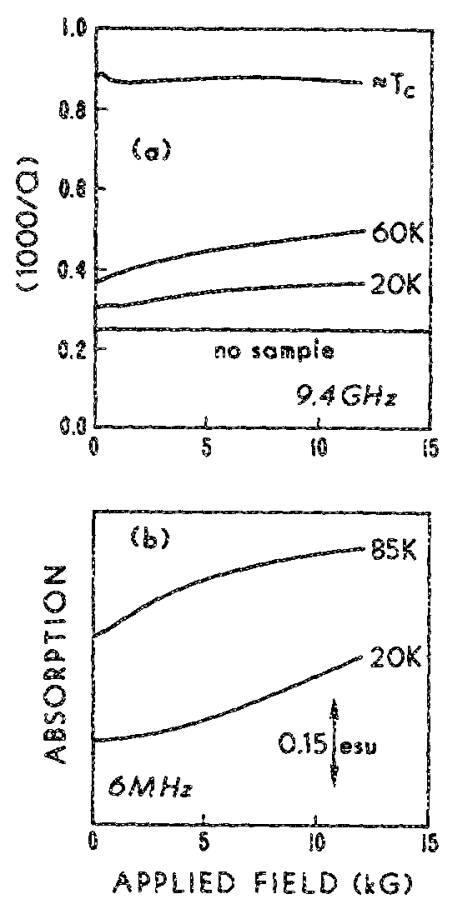

FIG. 9. (a) The inverse quality factor ( $1 / Q)$ of the microwave cavity containing a sample of $\mathrm{X}_{1} \mathrm{Ba}_{2} \mathrm{Cu}_{3} \mathrm{O}_{y}$ (see Fig. 1) at three temperatures. The quality factor $(Q)$ was determined by measuring the half width of the cavity resonance and includes contributions for the cavity walls as well as from the sample. (b) The broad component of the modulated absorption at $5 \mathrm{MH} z$ in $\mathrm{Y}_{1} \mathrm{Ba}_{2} \mathrm{Cu}_{3} \mathrm{O}_{y}$. 
observed modulated ac absorption below approximately 20 $G$ appears to exclude contributions from the critical state due to Abrikosov fuxons.

The evidence indicates that the majority of the modulated ac absorption originates from the individual superconducting grains. Blazey et al. ${ }^{33}$ report a revealing set of measurements of the ac absorption in a single crystal of $\mathrm{Y}_{1} \mathrm{Ba}_{2} \mathrm{Cl}_{3} \mathrm{O}_{y}$ which has a great deal of structure below $10 \mathrm{G}$ and is highly anisotropic. The shape and magnitude of the low-field ac absorption [Fig. 2(a)] is the same in a ceramic piece and a powder ground from the ceramic. The anisotropic ac absorption in a single grain taken from a ceramic piece (Fig. 7 ) is an additional confirmation that the absorption takes place within each grain or at its surface.

Ordinary surface impedance or "skin effect" ac absorption has already been discussed. The modulated absorption signal at large fields $(100 \mathrm{G}$ to $1.2 \mathrm{~T})$ is consistent with the expected behavior due to the surface impedance. ${ }^{16}$ To confirm this, we measured the width of the cavity dip (Q) of the microwave cavity with the superconducting sample in place. Figure 9 shows the cavity dissipation $(1 / Q)$ at three different temperatures for fields from 0 to $1.2 \mathrm{~T}$. At $T_{c}$, the sample is normal and the $Q$ shows no dependence (within experimental error) on the applied magnetic field. At lower temperatures the ac absorption due to the surface impedance is smallest at zero field and increases as the magnetic field penetrates the sample. Since the magnetic field penetration depth exceds $1000 \AA$ in the materials while the superconductor coherence length is less than $50 \AA$, the superconducting state should not be sensitive to the grain surfaces.

The observed modulated ac absorption is also distinct from the generally observed peak in the imaginary part of the magnetic susceptibility which is generally observed just below $T_{c}$. This peak in the magnetic ac absorption is due to inductive coupling of the induced supercurrents to guasinar ticle currents. ${ }^{34}$

\section{พ1. SUMAMARY}

The macroscopic ac magnetic susceptibility in the high transition temperature superconductors exhibits a large field-modulated absorption even at frequencies well below the superconducting energy gap and at temperatures below the superconducting transition temperature. The observed modulated absorption is sensitive to dc magnetic fields of a few Gauss and deereases with increasing frequency from 2 $\mathrm{MHz}$ to $9.4 \mathrm{GHz}$. The modulated absorption is characteristic of individual grains of superconducting material. Microwave rectification in ceramic samples of $\mathrm{Y}_{1} \mathrm{Ba}_{2} \mathrm{Cu}_{3} \mathrm{O}_{y}$ has similar characteristics to the modulated absorption and may be due to ac Josephson rectification. Several mechanisms are proposed but further theoretical modeling and experimentation is necessary before a complete model is possible.

\section{ACKNOWLEDGMENTS}

The $\mathrm{La}_{1,85} \mathrm{Sr}_{0.15} \mathrm{CuO}_{4}$ and $\mathrm{BPB}$ samples were provided by Z. C. Chen. This work was supported in part by the National Science Foundation under Grant No. DMR-8615217.
J. G. Becinorz and K. A. Müler, Z. Phys. B 64, 189 (1986); M. K. Wu, I. R. Ashburn, C. I. Torng, P. H. Hor, R. L. Meng, L. Gao, Z. J. Huang, Y. Q. Wang, and C. W. Chu, Phys. Rev. Lett. 58, 908 (1987); H. Maeda, Y. Tanaka, M. Fukutomi, and T. Asano, Jpn. J. Appl. Phys. P4. 227, L209 (1988).

'S. S. P. Parkin, V. Y. Lee, E. M. Engler, A. I. Nazzal, T. C. Huang, G. Gorman, R. Savoy, and R. Beyers, Phys. Rey, Lett. 60, 2539 (1988).

${ }^{3}$ R. N. Bhargava, S. R. Herko, and W. N. Osbortie, Phys. Rev. Lett. 39; 1468 (1987); J. T. Chen, L. E. Wenger, C. J. MeEwan, and E. M. Logothetis, Phys. Rev. Lert. 38, $1972(1987)$.

${ }^{4}$ R. J. Cava, B. Batlogg, R. B. van Dover, D. W. Mirphy, S. Sunshine, T. Siegrist, J. P. Remeika, E. A. Rietman, S. Zahurak, and G. P. Espinosa, Phys. Rev. Lett. 58, 1676 (1987).

${ }^{5}$ T. Ekino and J. Aximitsu, Jpn. J. Appl. Phys. 26, E452 (1987).

${ }^{6}$ A. Th. A. M. de Waele, R. T. M. Smokers, R. W. Van der Heijlen, K. Kadowaki, Y. K. Huang, M. van Sprang, and A. A. Menovsky, Phys. Rev. B 35, 8858 (1987); X. F. Meng, Y. D. Dai, H. M. Jiang, X. M. Ren, Y. Zhang, M. X. Yan, Y. C. Du, I. C. Mao, X. W. Wu, and G. J. Cui, Solid State Commun. 63, 853 (1987).

R. Durny, I. Hautala, S. Ducharme, B. Lee, O. G. Symko, P. C. Taylor, and D. J. Zheng, Phys. Rev. B 36, 2361 (1987).

${ }^{8}$ S. Ducharme, R. Durny, J. Hautala, O. G. Symko, P. C. Taylor, and S. Kulkarni, Mater. Res. Soc. Symp. Proc. 99, 845 (1988).

"S. V. Bhat, P. Ganguly, T. V. Ramakrishnan, and C. N. R. Rao, J. Phys. C 20, L559 (1987); K. N. Shrivastava, J. Phys. C 20, L789 (1987); M. D. Sastry, A. G. I. Dalvi, Y. Babu, R. M. Kadam, J. V. Yakhmi, and K. M. Iyer, Nature 330, 49 (1987); K. Khachaturyan, E. R. Weber, P. Tejedor, A. M. Stacy, and A. M. Portis, Phys. Rev. B 36, 8309 (1987).

${ }^{10} \mathrm{C}$. Rettori, D. Davidov, I. Belaish, and I. Felner, Phys. Rev. 36,4028 (1987).

"K.W. Blazey, K. A. Müller, J. G. Bednorz, W. Berlinger, G. Amorett, E. Buluggiu, A. Vera, and F. C. Matacotta, Phys, Rev. B 36, 7241 (1987).

iz. F. Kim, J. Bohandy, K. Mooriani, and F. J. Adrian, J. Appl. Phys. 63, 2029 (1988); J. Bohandy, J. Suter, B. F. Kim, K. Moorjani, and F. J. Adrian, Appl. Phys Lett. 51, 2161 (1987).

${ }^{13}$ A. M. Portis, K. W. Blazey, X. A. Müler, and X. G. Hednorz, Europhys. Lett. $\$$, 467 (1988); K. W. Blazey, A. M. Portis, and J. G. Bednorz, Solid State Commun. 65, 1153 (1988).

¿A. M. Pakulis and T. Osada, Phys. Rev. 13 37, 5940 (1988).

${ }^{15}$ M. Poirier, G. Quirion, K. R. Poeppelmeier, and J. P. Thiel, Phys. Rev. B 36, 3906 (1987); W. P. Beyerman, B. Alavi, and G. Grüner, Phys. Rev. B 36, 8826 (1987); E. J. Pakulis and T. Osada, Phys. Kev. B 37, 5940 (1988).

is. Sridhar, C. A. Shiffman, and H. Handeh, phys. Rev. B 36, 2301 (1987).

${ }^{17}$ A. T. Wigeratne, G. L. Dunifer, I. T. Chen, L. E. Wenger, and E. M. Logothetis, Phys. Rev. B 37, 615 (1988); J. Tateno and N. Masaki, Jpn. J. Appl. Phys. 26, L1654 (1987).

${ }^{18}$ R. Durny, S. Ducharme, J. Hautala, D. J. Zheng, O. O. Symko, P. C. Taylor, and S. Kulkarni, J. Opt. Soc. Am. B 6, 465 (1989); R. Durny, S. Ducharme, J. Hautala, O. G. Symko, P. C. Taylor, and S. Kulkarni, Mater. Res. Soc. Symp. Proc. 99, $849(1988)$.

${ }^{1}$ T. K. Worthington, W. J. Gallagher, and T. R. Dinger, Phys. Rev. Lett. 59, $1160(1987)$.

20M. Hervieu, B. Domenges, C. Michel, G. Heger, J. Provost, and B. Raveru, Phys. Rev. B 36, 3920 (1987).

${ }^{21}$ J. G. Bednorz, M. Takashige, and K. A. Müller, Europhys. Letr. 3, 379 (1987); K. A. Müller, M. Takashige, and 3. G. Bednorz, Phys. Rev. Lett. 58, 1143 (1987); M. Takashige, K. A. Mïller, and J. G. Bednorz, Ipn. J. Appl. Plyss. 26, Suppl. 26-3, 1101 (1987).

${ }^{22}$ M. Tuominen, A. M. Goldman, and M. L. Mecartney, Phys. Rev. B 3 ?, $548(1988)$.

${ }^{23}$ A. C. Mota, A. Pollini, P. Visani, E. A. Müller, and J. G. Bednorz, Phys. Rev. B 36, 4011 (1987).

${ }^{24}$ R. Durny, S. Ducharme, J. Hautala, D. J. Zheng, O. G. Synko, P. C. Taylor, and S. Kulkami (unpublished).

${ }^{25} \mathrm{M}$. Tinkham, Introduction to Supercondutivity (Krieger, New York, 1975), pp. 196-201.

${ }^{26} \mathrm{C}$. Ebner and D. Stroud, Phys. Rev. B 31, 165 (1985).

${ }^{27} \mathrm{G}$. Deutscher and X. A. Minler, Phys. Rev. Lett. 59, 1745 (1987).

2*. G. de Gennes, Superconductivity of Metals and Alloys (Bunjamin, New York, 1969).

${ }^{29}$ R. Durny, J. Hautala, S. Ducharme, D. J. Theng, O. G. Symko, P. C. Taylor, and S. Kulkarni, in the First Latin-American Conference on High Temperature Supercondictivity, Vol. 9 of Progress in High Temperature 
Superconductivity, edited by R. Nicolsky, R. A. Barrio, O. F. delima, and R. Escudero (World Scientific, Singapore, 1988), p. 343; O. G. Symko, D. J. Zheng, R. Durny, S. Ducharme, and P. C. Taylor, Phys. Lett. A 134, 72 (1988).

${ }^{30}$ A. M. Portis and K. W. Blazey, Solid State Commun. 68, 1097 (1988).

${ }^{31}$ Y. Yeshurun and A. P. Malozemof, Phys. Rev, i.ett. 60, 22012 (1988).
32Y. B. Kim and M. J. Stephen, in Superconductivity, edited by R. D. Parks (Dekker, New York, 1969).

${ }^{33} \mathrm{~K}$. W. Blazey, A. M. Portis, K. A. Müller, and F. H. Hoitzberg, Europhys. Lett. 6, 457 (1988).

${ }^{3.1}$ E. Poltrak and B. Fisher, Phys. Rev. B 36, 5586 (1987); T. Ishida and H. Mazakî, Jpn. J. Appl. Phys. 26, L1296 (1987). 